CEAB 95/9-13

NDA-FP-22

OCHA-PP-66

hep-th/9511167

August 1995

\title{
Quantum effects of stringy and membranic nature for the swimming of micro-organisms in a fluid
}

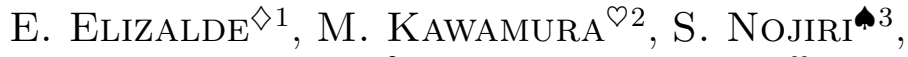 \\ S.D. Odintsovert and A. Sugamoto 9 官 \\ $\diamond$ Dipartimento di Fisica, Università degli Studi di Trento, Italia \\ and Center for Advanced Studies CEAB, CSIC, Camí de Santa Bàrbara, 17300 Blanes, \\ Spain \\ $\odot$ Department of Physics, Ochanomizu University \\ 1-1, Otsuka 2, Bunkyo-ku, Tokyo, 112, Japan \\ A Department of Mathematics and Physics, National Defence Academy \\ Yokosuka, 239, Japan \\ a Tomsk Pedagogical Institute, 634041 Tomsk, Russia, \\ and Department ECM, Faculty of Physics, University of Barcelona, \\ Diagonal 647, 08028 Barcelona, Spain
}

\begin{abstract}
The static potential is investigated in string and membrane theories coupled to $U(1)$ gauge fields (specifically, external magnetic fields) and with antisymmetric tensor fields. The explicit dependence of the potential on the shape of the extended objects is obtained, including a careful calculation of the quantum effects. Noting the features which are common to the dynamics of strings and membranes moving in background fields and to the swimming of micro-organisms in a fluid, the latter problem is studied. The Casimir energy of a microorganism is estimated, taking into account the quantum effects and the backreaction from the outside fluid.

\footnotetext{
${ }^{1}$ E-mail: eli@zeta.ecm.ub.es

${ }^{2}$ E-mail: masako@phys.ocha.ac.jp

${ }^{3}$ E-mail: nojiri@cc.nda.ac.jp

${ }^{4}$ E-mail: sergei@ecm.ub.es.

${ }^{5}$ E-mail: sugamoto@phys.ocha.ac.jp
} 


\section{Introduction.}

The very basic problem in Biology of the description of the swimming of micro-organisms has been analysed from a gauge theoretical point of view by Shapere and Wilczek [1, 2] and from a string (or membrane) theoretical viewpoint in Refs. [5]-[5]. The surface of a microorganism has one (resp. two) dimensions in a two (resp. three) dimensional fluid, and it can be regarded as a special kind of string (or membrane). In particular, the shapes of the surfaces of ciliate and flagellate in two dimensions are similar to those of closed and open strings, respectively. Owing to the backreaction of the velocity fields, there appear interactions between these strings, which are similar to the interaction mediated by an antisymmetric tensor field [6] and others.

In the case of micro-organisms, the string (i.e., the surface of the micro-organism) couples to the velocity fields in a special way. Furthermore the velocity fields can be regarded as gauge fields in a $U(1)$ gauge theory. By integrating the velocity fields, Coulomb-like interactions are created between the strings. Therefore, it is an interesting issue to consider in a unified fashion the two problems, namely that of string and membrane theories coupled with various external fields, including the $U(1)$ gauge field, and the swimming problem of micro-organisms inside a fluid, and thus obtain the static potential and the Casimir energy corresponding to the last one from our knowledge of the first, general theory.

The paper is organized as follows. In sections 2 and 3 the theoretical background for the analysis will be provided. In particular, in Sect. 2 we obtain the static potential for a bosonic string in a magnetic field. In Sect. 3 the formulas for the case of the bosonic membrane in a magnetic field are derived. In section 4, the estimation of the static potential in the string theory coupled to an antisymmetric field is briefly summarized. In Sect. 5, we proceed with the application of the theory to the description of the swimming of micro-organisms. In particular, we consider in detail the quantum (thermal) effects - after adding the potential term - and we show explicitly the appearance of a kind of Casimir energy.

Of course, there are some differences between the usual string and the micro-organism considered as such. The energy of the usual string is proportional to its length but the stable shape of ciliate should be a circle with a finite radius and the flagellum is not elastic (the length is not changable). Another difference is that the apparent divergence in the zero-point energy is not an ultra-violet but an infrared one. The reason for this is the fact that the efficiency of the translation or rotation of the micro-organism becomes better for higher modes [2].

\section{Static potential for the bosonic string in a magnetic field.}

We will consider here a bosonic string theory interacting with a magnetic field [7]. The corresponding action has the following form (in the Euclidean notation)

$$
S=\frac{k}{2} \int d^{2} \xi \sqrt{g}\left[\partial_{i} X^{\mu} \partial^{i} X_{\mu}+2 A_{p}(X) \partial_{i} X^{p} \partial^{i} X^{1}\right],
$$

being $i=0,1$ and where the string coordinates $X^{\mu}$ describe a four-dimensional space-time ( $\mu=0,1, p$, with $p=2,3)$. The parametrization of the string coordinates is

$$
X_{c l}^{0}=\xi_{0}, \quad X_{c l}^{1}=\xi_{1}, \quad 0 \leq \xi_{0} \leq t, 0 \leq \xi_{1} \leq R,
$$

with the corresponding quantum fluctuations vanishing at the boundary. This represents a once-wrapped torus. 
The semi-classical quantization of the theory is conveniently considered in the background gauge

$$
X^{0}=X_{c l}^{0}, \quad X^{1}=X_{c l}^{1},
$$

in which there are no Faddeev-Popov ghosts. At the one-loop level, the only quantum fluctuations which contribute to the path integral are those corresponding to $X^{3}$ and $X^{4}$ [8, 9].

The following form will be chosen for the vector field:

$$
A_{p}(X)=-\frac{1}{2} f \epsilon_{p m} X^{m}
$$

Note that $f$ in Eq. (团) plays the role of a magnetic field. In principle one can consider the more general situation when $D>4$, by writing the vector field in a block-diagonal form, being each block described as in (4).

The static potential or Casimir energy at one-loop can be obtained as follows:

$$
V=-\lim _{t \rightarrow \infty} \frac{1}{t} \ln \int \mathcal{D} X^{m} e^{-S(t)}
$$

where

$$
S(t)=k \int_{0}^{t} d \xi_{0} \int_{0}^{R} d \xi_{1}\left[1+\frac{1}{2} \partial_{i} X^{m} \partial^{i} X_{m}-\frac{f}{2}\left(X^{3} \partial_{1} X^{2}-X^{2} \partial_{1} X^{3}\right)\right] .
$$

Performing the integrations over $X^{2}$ and $X^{3}$, by taking into account the fact that for those fields the classical background is zero, and by satisfying the standard periodic boundary conditions, one obtains

$$
V=k R+\frac{1}{2} \operatorname{Tr} \ln \left(\begin{array}{cc}
-\square & f \partial_{1} \\
-f \partial_{1} & -\square
\end{array}\right)=k R+\frac{1}{2} \ln \operatorname{det}\left(\square^{2}+f^{2} \partial_{1}^{2}\right),
$$

where $\square=\partial_{0}^{2}+\partial_{1}^{2}$. Observe that when we write the operators $\operatorname{Tr} \ln$ and $\ln$ det we understand that the factor $1 / t$ is included. In absence of the magnetic field, $f=0$, the calculation of $\ln$ det $\square$ yields $[8,9]$

$$
V(R)=k R-\frac{\pi}{12 R} .
$$

Notice again that this is the one-loop result. In the case of a non-vanishing magnetic field $f \neq 0$, using the zeta-function method [23, 24] (for review, see [10]) we get [11]:

$$
V(R)=k R-\frac{1}{2} \zeta_{A}^{\prime}(0)
$$

where

$$
\begin{aligned}
\zeta_{A}(s) & =\int d^{2} p\left(p \cdot p+f^{2} p_{1}^{2}\right)^{-s}=\int_{0}^{\infty} d p \sum_{n=1}^{\infty}\left[p^{2}+\frac{\left(1+f^{2}\right) n^{2}}{R^{2}}\right]^{-s} \\
& =\frac{1}{\Gamma(s)} \int_{0}^{\infty} d t t^{s-1} \sum_{n=1}^{\infty} e^{-\left(1+f^{2}\right) n^{2} t / R^{2}} \int_{0}^{\infty} d p e^{-p^{2} t}=-\frac{\sqrt{\pi} \Gamma(s-1 / 2) R^{2 s-1}}{2 \Gamma(s)\left(1+f^{2}\right)^{s-1 / 2}} \zeta(2 s-1) .
\end{aligned}
$$


By taking now the derivative at $s=0$, this yields the result

$$
-\zeta_{A}^{\prime}(0)=\int d^{2} p \ln \left(p \cdot p+f^{2} p_{1}^{2}\right)=\int_{0}^{\infty} d p \sum_{n=1}^{\infty} \ln \left(p^{2}+\frac{\left(1+f^{2}\right) n^{2}}{R^{2}}\right)=-\frac{\pi \sqrt{1+f^{2}}}{6 R} .
$$

Finally, for the static potential we obtain

$$
V(R)=k R-\frac{\pi \sqrt{1+f^{2}}}{12 R} .
$$

The stronger the magnetic field is, the bigger is the quantum correction to the static potential.

\section{Bosonic membrane with a magnetic field.}

Let us now consider the action corresponding to the Howe-Tucker bosonic membrane [12. In the Euclidean notation, it is given by the action

$$
S=\frac{k}{2} \int d^{3} \xi \sqrt{\gamma}\left(\gamma^{i j} \partial_{i} X^{\mu} \partial^{j} X_{\mu}-1\right)
$$

where $k$ is the membrane tension. Here $\xi^{i}, i=0,1,2$, denote the world-volume coordinates, $\gamma^{i j}$ the world-volume metric, and $X^{\mu}, \mu=0,1, \ldots, d-1$, denote the space-time coordinates, with metric $g^{\mu \nu}$. In particular, we will consider here the situation corresponding to $d=5$ space-time coordinates, that is $\mu=0,1,2, p$, with $p=3,4$.

The classical solution for the theory (14) of toroidal form is given by

$$
X_{c l}^{0}=\xi_{0}, \quad X_{c l}^{1}=\xi_{1}, \quad X_{c l}^{2}=\xi_{2},
$$

and $\left(\xi_{1}, \xi_{2}\right) \in R \equiv\left[0, A_{1}\right] \times\left[0, A_{2}\right]$. It represents a once-wrapped torus.

For the semi-classical quantization of the theory, it is convenient to consider it in the background gauge

$$
X^{0}=X_{c l}^{0}, \quad X^{1}=X_{c l}^{1}, \quad X^{2}=X_{c l}^{2} .
$$

In this gauge there are again no Faddeev-Popov ghosts, in other words, quantum fluctuations for the three space-time coordinates above should not be taken into account in the calculation of the corresponding path-integral. For $X^{p}, p=3,4$, there are no classical fields. They represent the fluctuations that are completely quantum, and satisfy the standard periodic boundary conditions corresponding to the torus.

We can now consider the interaction of the bosonic membrane with a vector field:

$$
S_{I}=\frac{k}{2} \int d^{3} \xi \sqrt{\gamma}\left[2 A_{p}(X) \partial_{i} X^{p}\left(\partial^{i} X^{1}+\partial^{i} X^{2}\right)\right] .
$$

The form of the vector field can be conveniently chosen to be the following

$$
A_{p}(X)=-\frac{1}{2} f \epsilon_{p m} X^{m}
$$

where $f$ plays the role of the magnetic field. It is interesting to observe that the interaction of a string theory with a magnetic field can be considered in a similar way (see the previous section). 
The static potential can be obtained as

$$
V=-\lim _{t \rightarrow \infty} \frac{1}{t} \ln \int \mathcal{D} X^{m} e^{-S(t)},
$$

where

$$
S(t)=k \int_{0}^{t} d \xi_{0} \int_{R} d^{2} \xi\left[1+\frac{1}{2} \partial_{i} X^{m} \partial^{i} X_{m}+\frac{f}{2} X^{3}\left(\partial_{1} X^{4}+\partial_{2} X^{4}\right)-\frac{f}{2} X^{4}\left(\partial_{1} X^{3}+\partial_{2} X^{3}\right)\right] .
$$

Now, one can calculate the path integral over $X^{m}$. In the one-loop approximation, we get

$$
V=k A_{1} A_{2}+\frac{1}{2} \operatorname{Tr} \ln \left(\begin{array}{cc}
-\square & f\left(\partial_{1}+\partial_{2}\right) \\
-f\left(\partial_{1}+\partial_{2}\right) & -\square
\end{array}\right)
$$

that is,

$$
V=k A_{1} A_{2}+\frac{1}{2} \ln \operatorname{det}\left[\square^{2}+f^{2}\left(\partial_{1}+\partial_{2}\right)^{2}\right]
$$

where $\square=\partial_{0}^{2}+\partial_{1}^{2}+\partial_{2}^{2}$ is here the three-dimensional d'Alembertian.

As before, in the general case of a non-vanishing magnetic field, $f \neq 0$, using the zetafunction method [10] we obtain [11]:

$$
V\left(A_{1}, A_{2}\right)=k A_{1} A_{2}-\frac{1}{2} \zeta_{A}^{\prime}(0)
$$

where the zeta function is now given by

$$
\begin{aligned}
\zeta_{A}(s) & =\int d^{2} p\left[p \cdot p+f^{2}\left(p_{1}+p_{2}\right)^{2}\right]^{-s} \\
& =\int_{0}^{\infty} d p \sum_{n_{1}, n_{2}}\left[p^{2}+\left(1+f^{2}\right)\left(\frac{n_{1}^{2}}{A_{1}^{2}}+\frac{n_{2}^{2}}{A_{2}^{2}}\right)+2 f^{2} \frac{n_{1} n_{2}}{A_{1} A_{2}}\right]^{-s} . \\
& =\frac{\sqrt{\pi} \Gamma(s-1 / 2)}{4 \Gamma(s)} F\left(s-\frac{1}{2} ; \frac{1+f^{2}}{A_{1}^{2}}, \frac{2 f^{2}}{A_{1} A_{2}}, \frac{1+f^{2}}{A_{2}^{2}}\right),
\end{aligned}
$$

The function $F(s ; a, b, c)$ is the analytical continuation of the general Epstein zeta function in two dimensions, which is given by the celebrated Chowla-Selberg formula [13] (for extensions of this formula to inhomogeneous cases and truncated sums, see [14, [15])

$$
\begin{gathered}
F(s ; a, b, c)=2 \zeta(2 s) a^{-s}+\frac{2^{2 s} \sqrt{\pi} a^{s-1}}{\Gamma(s) \Delta^{s-1 / 2}} \Gamma(s-1 / 2) \zeta(2 s-1)+\frac{2^{s+5 / 2} \pi^{s}}{\Gamma(s) \Delta^{s / 2-1 / 4} \sqrt{a}} \\
\times \sum_{n=1}^{\infty} n^{s-1 / 2} \sigma_{1-2 s}(n) \cos (n \pi b / a) K_{s-1 / 2}\left(\frac{\pi n \sqrt{\Delta}}{a}\right) .
\end{gathered}
$$

where

$$
\sigma_{s}(n) \equiv \sum_{d \mid n} d^{s},
$$


this sum extending over all divisors $d$ of $n$. In the general theory dealing with the homogeneous case, one assumes that $a, c>0$ and that the discriminant

$$
\Delta=4 a c-b^{2}>0
$$

(see [13). We obtain

$$
\Delta=\frac{4}{A_{1}^{2} A_{2}^{2}}\left(1+f^{2}+f^{4}\right)
$$

and for the zeta function

$$
\begin{aligned}
\zeta_{A}(s) & =\frac{\sqrt{\pi}}{2} \frac{\left(1+f^{2}\right)^{1 / 2-s}}{A_{1}^{1-2 s}} \frac{\zeta(2 s-1)}{\Gamma(s)}+\frac{2^{2 s-3} \pi A_{1}^{3-2 s}}{A_{1}^{3-2 s} \Delta^{s-1}} \frac{\zeta(2 s-2)}{s-1} \\
& +\frac{(2 \pi)^{s} A_{1}}{\Gamma(s) \Delta^{(s-1) / 2} \sqrt{1+f^{2}}} \sum_{n=1}^{\infty} n^{s-1} \sigma_{2-2 s}(n) \cos \left(2 \pi n \frac{A_{1}}{A_{2}} \frac{f^{2}}{1+f^{2}}\right) K_{s-1}\left(\frac{\pi n A_{1}^{2} \sqrt{\Delta}}{1+f^{2}}\right) .
\end{aligned}
$$

The good convergence properties of expression (28) were very much prised by Chowla and Selberg. They render the use of the formula (32) above quite simple. In fact, the two first terms are just nice while the last one (impressive in appearence) is very quickly convergent in the real case, and thus absolutely harmless in practice. Only a few first terms of the three series of Bessel functions in (32), need to be calculated, even if one demands good accuracy.

By taking now the derivative at $s=0$, this zeta function yields the following result for the effective potential

$$
\begin{aligned}
V\left(A_{1}, A_{2}\right)= & k A_{1} A_{2}-\frac{\pi \sqrt{1+f^{2}}}{12 A_{1}}+\frac{\pi \zeta^{\prime}(-2) A_{1}^{3} \Delta}{8\left(1+f^{2}\right)^{3 / 2}} \\
& -\frac{A_{1} \sqrt{\Delta}}{\sqrt{1+f^{2}}} \sum_{n=1}^{\infty} n^{-1} \sigma_{2}(n) \cos \left(2 \pi n \frac{A_{1}}{A_{2}} \frac{f^{2}}{1+f^{2}}\right) K_{s-1}\left(\frac{\pi n A_{1}^{2} \sqrt{\Delta}}{1+f^{2}}\right) .
\end{aligned}
$$

As one can see, the $f$-dependence of the static potential is much more complicated, as compared with string theory.

\section{Static potential for the string coupled to an antisymmetric tensor field.}

This case has been studied in [6]. After integrating over $A_{i j}$ and $\phi$ in order to obtain an effective theory for the closed bosonic string, exhibiting a Coulomb-like interaction term (compare with Ref. [22]), we get

$$
\begin{aligned}
S= & \int d^{2} \xi \sqrt{g}\left[\frac{1}{2} G_{\mu \nu}(X) g^{i j} \partial_{i} X^{\mu} \partial_{j} X^{\nu}\right]+S_{i n t}, \\
S_{i n t}= & \int d^{2} \xi d^{2} \xi^{\prime}\left[c_{1} e^{2} \sigma_{\mu \nu}(\xi) \sigma^{\mu \nu}\left(\xi^{\prime}\right)+c_{2} \zeta^{2} R^{(2)^{2}}\right] V\left(\left|x-x^{\prime}\right|\right), \\
& \sigma_{\mu \nu}(\xi)=\epsilon^{i j} \partial_{i} X^{\mu} \partial_{j} X^{\nu}, \quad V\left(\left|x-x^{\prime}\right|\right)=\frac{1}{\left|x(\xi)-x\left(\xi^{\prime}\right)\right|^{2}+a^{2}},
\end{aligned}
$$


being $c_{1}$ and $c_{2}$ non-essential numerical constants which can be choosen to be equal to 1 . The static potential in string theory is an interesting magnitude in connection with the possible applications of string theory to QCD. This fact was realized long ago [20], and a calculation of the static potential in different string models has been carried out explicitly in Refs. [8, 9], and in particular for the rigid string [21] in Refs. [16]-[18]. It has been pointed out in those works that the leading corrections to the static potential have a universal character [9]. In the applications of the static potential to QCD one can usually choose the Wilson loop $C$ to be a rectangle on the plane, of length $T$ and width $R$ (with $T>>R$ ). Then, the loop expectation value can be found as follows [19]:

$$
W[C] \sim \exp [-T V(R)] .
$$

The explicit one-loop calculation for a standard bosonic string gives:

$$
V(R)=k R+\frac{D-2}{2} \operatorname{Tr} \ln \square
$$

where $k$ is again the string tension and $\square$ the two-dimensional d'Alembertian in the space of topology $R \times S^{1}$. Using the zeta-function regularization procedure to calculate (40) one obtains the well-known result [9, 8, 11] (for simplicity, we consider the case $D=4$ )

$$
V(R)=k R-\frac{(D-2) \pi}{24 R} .
$$

The calculation to one-loop of the static potential (40) taking into account the Coulomb-like term induced by the antisymmetric tensor fields, as in (37), is quite non trivial since the integration over the $X^{\mu} \mathrm{s}$ is not Gaussian. The natural way to consider the integration is by decomposing the variables as follows:

$$
X^{\mu}(\xi)=X_{0}^{\mu}(\xi)+X_{1}^{\mu}(\xi)
$$

where $X_{0}^{\mu}(\xi)$ is a background variable, which is a linear function of $\xi$ satisfying the field equations

$$
X_{0}^{\mu}(\xi)=c_{i}^{\mu} \xi^{i}, \quad \eta_{\mu \nu} c_{i}^{\mu} c_{j}^{\nu}=\eta_{i j}
$$

being the $c_{i}^{\mu}$ some constants. The expansion of Eq. (37) up to second order on the fluctuations $X_{1}^{\mu}(\xi)$ can be performed in the same way as it was done in Ref. [22]. After the subsequent functional Gaussian integration over the $X_{1}^{\mu}$ s, we obtain the static potential

$$
V(R)=k R+\frac{D-2}{2} \int d p \sum_{n=1}^{\infty} \ln \left[\frac{p^{2}}{2}+2 e^{2} p^{2} \int d^{2} \xi \frac{e^{i p \cdot \xi}}{\xi \cdot \xi+a^{2}}+4 e^{2} \int d^{2} \xi \frac{e^{i p \cdot \xi}-1}{\left(\xi \cdot \xi+a^{2}\right)^{2}}\right] .
$$

Notice that the notation has been simplified somehow, because in (44) it must be understood that the double integration over $d^{2} \xi$ is in fact a single integration on the first coordinate $\xi_{1}$ and an infinite sum (one of the spatial coordinates corresponds to the torus), exactly as in the case of the first integration (over $p$ and $n$ ). After some work, one obtains [6]

$$
\begin{aligned}
V(R)= & k R-\frac{(D-2) \pi}{24 R}+\frac{D-2}{2} \int d p \sum_{n=1}^{\infty} \ln \left\{4 \pi e^{2} \sum_{m=1}^{\infty} \frac{e^{i n m / R} e^{-p \sqrt{m^{2}+a^{2}}}}{\sqrt{m^{2}+a^{2}}}\right. \\
& \left.+\frac{4 \pi e^{2}}{p^{2}+n^{2} / R^{2}} \sum_{m=1}^{\infty}\left[\frac{e^{i n m / R} e^{-p \sqrt{m^{2}+a^{2}}}\left(1+p \sqrt{m^{2}+a^{2}}\right)-1}{\left(m^{2}+a^{2}\right)^{3 / 2}}-\frac{1}{2\left(m^{2}+a^{2}\right)^{3 / 2}}\right]\right\} .
\end{aligned}
$$


No approximation is involved in Eq. (45). However, this expression is quite complicated and to proceed further one has to do some approximation, valid in the limit when $a$ is big $(a>R)$. The first approximation in $1 / a$, obtained by keeping just the leading terms of the final result, yields

$$
V(R) \simeq k R-\frac{(D-2) \pi}{24 R}\left[1-\frac{6 \pi e^{2} R^{2}}{a^{3}}\left(\gamma+2 \operatorname{Sinint}(1)-\pi+e^{-a /(R)}\right)-\frac{24 \pi e^{2}}{a\left(e^{a /(R)}-1\right)}\right]
$$

where $\gamma$ is Euler's constant and Sinint the standard sinus integral (Sinint $(1)=0.946083$ ). (Note that in this expression we have kept a couple of terms which are representative of the asymptotically smaller contributions to the effective potential that can be dismissed completely in this approximation.) The dependence on $a$ and $R$ could have been ascertained by dimensional reasons. Numerically, the result is:

$$
V(R)=k R-\frac{(D-2) \pi}{24 R}\left\{1-\frac{e^{2} R^{2}}{a^{3}}\left[25.3416+\mathcal{O}\left(\frac{1}{a}\right)\right]\right\} .
$$

Having at hand this result for the effective potential, we can now study in some detail the contribution of the antisymmetric fields to the static potential. From (48) we see immediately that the static potential is given by expression (41) with a renormalized string tension, namely

$$
V(R)=k_{R} R-\frac{(D-2) \pi}{24 R}
$$

where

$$
k_{R} \simeq k+3.3172(D-2) \frac{e^{2}}{a^{3}} .
$$

Hence, we observe that when the radius $R$ equals $R_{c}, R_{c}^{2}=(D-2) \pi /\left(24 k_{R}\right)$, it turns out that $V\left(R_{c}\right)=0$. The appearance of this critical radius, $R_{c}$ indicates very probably - as in more complicated string models - that the quasi-static string picture ceases to be valid there, what has been interpreted in Ref. [8] (using a different string model as example) to be a signal for a phase transition. From this point of view, it seems natural to interprete the effect of the antisymmetric fields in the static potential as a renormalization of the string constant $k$ (a one-loop correction to the classical potential), what produces a change in the value of the critical radius $R_{c}$, as compared with the one that it has in the case when there is no coupling with antisymmetric fields.

\section{Static potential and the Casimir effect for the micro-organisms.}

In this section, we will consider the backreaction of the velocity fields for the swimming of micro-organisms in a fluid, especially in a two-dimensional fluid. We use the canonical formalism for convienience and evaluate the zero point energy of the Hamiltonian. The formalism is, of course, equivalent to the path integral formalism in the previous sections and the obtained energy has a form similar to those of the static potentials in the previous sections.

For the case of micro-organisms' swimming, the Reynolds number is very small and the Navier-Stokes equation becomes linearized. Therefore, the equations of motion for the 
incompressible fluid are given here by

$$
\begin{aligned}
\nabla \cdot \mathbf{v} & =0 \\
\Delta \mathbf{v} & =\frac{1}{\eta} \nabla p \text { or } \Delta(\nabla \times \mathbf{v})=0 .
\end{aligned}
$$

Here $\mathbf{v}, p$ and $\eta$ are the velocity field, the pressure and the viscosity coefficient, respectively. In two dimensions, Eqs. (51) and (52) acquire the specific form

$$
\begin{aligned}
\partial_{z} v_{\bar{z}}+\partial_{\bar{z}} v_{z} & =0, \\
4 \eta \partial_{z} \partial_{\bar{z}} v_{\bar{z}} & =\partial_{\bar{z}} p, \\
4 \eta \partial_{z} \partial_{\bar{z}} v_{z} & =\partial_{z} p .
\end{aligned}
$$

It is an interesting point that the equations of motion (53) and (54) can be derived from the following QED-like Lagrangean in the Landau gauge:

$$
\mathcal{L}=2 \eta\left(\partial_{z} v_{\bar{z}}-\partial_{\bar{z}} v_{z}\right)^{2}-p\left(\partial_{z} v_{\bar{z}}+\partial_{\bar{z}} v_{z}\right)
$$

Furthermore, the Lagrangean (55) can be regarded as the entropy density of the fluid, what will be shown next.

According to any standard textbook on the theory of fluid dynamics 9 , one finds that the time-derivative of the fluid entropy $S$ is given by the expression

$$
\dot{S}=\int d V\left[\frac{\sigma_{i k}^{\prime}}{2 T}\left(\frac{\partial v_{i}}{\partial x_{k}}+\frac{\partial v_{k}}{\partial x_{i}}\right)-\frac{\mathbf{q} \cdot \operatorname{grad} T}{T^{2}}\right] .
$$

Here $\sigma_{i k}^{\prime}$ is the stress tensor (in three dimensions):

$$
\sigma_{i k}^{\prime}=\eta\left(\frac{\partial v_{i}}{\partial x_{k}}+\frac{\partial v_{k}}{\partial x_{i}}-\frac{2}{3} \delta_{i k} \frac{\partial v_{l}}{\partial x_{l}}\right)+\zeta \delta_{i k} \frac{\partial v_{l}}{\partial x_{l}}
$$

and $\mathbf{q}$ is the heat current density. If we assume that the temperature $T$ can be globally defined, $\operatorname{grad} T=0$, and that the fluid is incompressible (51), Eq. (56) can be rewriten as

$$
\dot{S}=\int d V \frac{\eta}{2 T}\left[\left(\frac{\partial v_{i}}{\partial x_{k}}-\frac{\partial v_{k}}{\partial x_{i}}\right)^{2}+p \frac{\partial v_{l}}{\partial x_{l}}+\frac{\partial}{\partial x_{i}}\left(v_{k} \frac{\partial v_{i}}{\partial x_{k}}\right)\right] .
$$

Here we have imposed the condition of incompressibility by means of the multiplier field $p$. The resulting expression is equivalent to the action of QED in the Landau gauge if we neglect the surface term.

Equation (58) tells us that the entropy of the fluid in two dimensions satisfying the boundary condition on the surface of the micro-organisms is given by

$$
\begin{aligned}
\dot{S}= & \frac{\eta}{2 T}\left[\frac{i}{2} \int d z d \bar{z}\left\{4\left(\partial_{z} v_{\bar{z}}-\partial_{\bar{z}} v_{z}\right)^{2}-p\left(\partial_{z} v_{\bar{z}}+\partial_{\bar{z}} v_{z}\right)\right\}\right. \\
& +\int_{0}^{2 \pi} d \sigma\left\{P(\sigma)\left(\dot{Z}(\sigma)-v^{z}(Z(\sigma), \bar{Z}(\sigma))\right)\right. \\
& \left.\left.+\bar{P}(\sigma)\left(\dot{\bar{Z}}(\sigma)-v^{\bar{z}}(Z(\sigma), \bar{Z}(\sigma))\right)\right\}\right]
\end{aligned}
$$

\footnotetext{
${ }^{6}$ We use here the notation of Landau and Lifschitz.
} 
Here $\sigma$ parametrizes the surface of the micro-organism and $Z(\sigma)$ expresses the shape of the surface, like a string coordinate. In the terminology of the previous sections

$$
X^{0}=t=\xi_{0}, \quad Z(t, \sigma)=X^{1}(t, \sigma)+i X^{2}(t, \sigma) \quad\left(\sigma=\xi_{1} / R\right)
$$

The second term in Eq. (59) gives the condition that there is no slipping between the surface of the micro-organism and the fluid.

By integrating over the velocity fields $v_{z}$ and $v_{\bar{z}}$, we obtain the following Coulomb-like interaction between the $P(\sigma)$ 's:

$$
\begin{aligned}
\dot{S}= & \frac{\eta}{2 T} \int_{0}^{2 \pi} d \sigma\{P(\sigma) \dot{Z}(\sigma)+\bar{P}(\sigma) \dot{\bar{Z}}(\sigma)\} \\
& +\int_{0}^{2 \pi} d \sigma \int_{0}^{2 \pi} d \sigma^{\prime}\left\{P(\sigma) P\left(\sigma^{\prime}\right) G^{z z}\left(Z(\sigma), Z\left(\sigma^{\prime}\right)\right)\right. \\
& +\bar{P}(\sigma) \bar{P}\left(\sigma^{\prime}\right) G^{\bar{z} \bar{z}}\left(Z(\sigma), Z\left(\sigma^{\prime}\right)\right) \\
& \left.+2 P(\sigma) \bar{P}\left(\sigma^{\prime}\right) G^{z \bar{z}}\left(Z(\sigma), Z\left(\sigma^{\prime}\right)\right)\right\}
\end{aligned}
$$

Here $G_{z z}\left(z, z^{\prime}\right), G_{\bar{z} \bar{z}}\left(z, z^{\prime}\right)$ and $G_{z \bar{z}}\left(z, z^{\prime}\right)$ are defined by

$$
\begin{aligned}
G_{z z}\left(z, z^{\prime}\right) & =\frac{1}{2 \pi} \frac{\left(z-z^{\prime}\right)^{2}}{\left|z-z^{\prime}\right|^{2}}, \\
G_{\bar{z} \bar{z}}\left(z, z^{\prime}\right) & =\frac{1}{2 \pi} \frac{\left(\bar{z}-\bar{z}^{\prime}\right)^{2}}{\left|z-z^{\prime}\right|^{2}}, \\
G_{z \bar{z}}\left(z, z^{\prime}\right) & =G_{\bar{z} z}\left(z, z^{\prime}\right) \\
& =\frac{1}{2 \pi} \ln \left|z-z^{\prime}\right|^{2} .
\end{aligned}
$$

In order to continue integrating over $P(\sigma)$ further, we can use the equation of motion, which is given by the variation of $S$ with respect to $P(\sigma)$ :

$$
0=\dot{\bar{Z}}(\sigma)+2 \int_{0}^{2 \pi} d \sigma^{\prime}\left\{P\left(\sigma^{\prime}\right) G^{z z}\left(Z(\sigma), Z\left(\sigma^{\prime}\right)\right)+\bar{P}\left(\sigma^{\prime}\right) G^{z \bar{z}}\left(Z(\sigma), Z\left(\sigma^{\prime}\right)\right)\right\}
$$

In the following, we consider the swimming motion of the micro-organism with cilia and we assume that the shape of the micro-organism is given by

$$
Z(\sigma)=R \mathrm{e}^{i \sigma}+\sum_{n \neq 0,1} \mathrm{e}^{i n \sigma} z_{n}, \quad\left(z_{n} \ll R\right)
$$

We now solve Eq. (62) with respect to $P(\sigma)$ to first order in $z_{n}$. Then we can approximate $G_{z z}\left(z, z^{\prime}\right)$ and $G_{z \bar{z}}\left(z, z^{\prime}\right)$ by

$$
\begin{aligned}
G_{z z}\left(z, z^{\prime}\right) & \sim-\frac{1}{2 \pi} \mathrm{e}^{i\left(\sigma+\sigma^{\prime}\right)} \\
G_{z z}\left(z, z^{\prime}\right) & \sim \frac{1}{2 \pi} \ln R\left|1-\mathrm{e}^{i\left(\sigma-\sigma^{\prime}\right)}\right| \\
& =\frac{1}{2 \pi}\left\{\ln R+\sum_{n=1}^{\infty} \frac{1}{n}\left(\mathrm{e}^{i n\left(\sigma-\sigma^{\prime}\right)}+\mathrm{e}^{-i n\left(\sigma-\sigma^{\prime}\right)}\right)\right\} .
\end{aligned}
$$


By expanding $P(\sigma): P(\sigma)=\sum_{n} \mathrm{e}^{i n \sigma} p_{n}$ and substituting it into Eq. (62), we obtain the following equation to first order in $z_{n}$ :

$$
0=\sum_{n \neq 0,1} \mathrm{e}^{i n \sigma} \dot{z}_{n}+2 \mathrm{e}^{i \sigma} p_{-1}+2 \sum_{n=1}^{\infty} \frac{1}{n}\left(\mathrm{e}^{i n \sigma} \bar{p}_{n}+\mathrm{e}^{-i n \sigma} \bar{p}_{-n}\right) .
$$

Then we find

$$
\begin{aligned}
p_{n} & =-\frac{|n|}{2} \dot{\bar{z}}_{-n}, \quad(n \neq 0,-1) \\
p_{-1} & =0 .
\end{aligned}
$$

Note that $p_{0}$ cannot be determined but, actually, they do not appear in Eq. (60). By substituting the solutions in Eq. (66), we obtain

$$
\dot{S}=-\frac{\pi \eta}{2 T} \sum_{n \neq 0,1}|n| \dot{z}_{n} \dot{\bar{z}}_{n}
$$

The "action" $S$ in Eq. (67) is proportional to the power of the micro-organism.

We now add the potential term which makes the shape of micro-organism to be a circle of radius $R$ :

$$
V=\lambda^{2} R \int_{0}^{2 \pi} d \sigma\left|Z(\sigma)-R \mathrm{e}^{i \sigma}\right|^{2}=2 \pi \lambda^{2} R \sum_{n \neq 0,1} \bar{z}_{n} z_{n} .
$$

Then the total "Lagrangean" $L$ is given by

$$
L=\frac{\pi \eta}{2 T} \sum_{n \neq 0,1}|n| \dot{z}_{n} \dot{\bar{z}}_{n}-\frac{2 \pi \lambda^{2} R}{T} \sum_{n \neq 0,1} \bar{z}_{n} z_{n} .
$$

If we set $z_{n}=x_{n}+i y_{n}$, the conjugate momenta corresponding to $x_{n}$ and $y_{n}$ are given by

$$
p_{n}^{x} \equiv \frac{\partial L}{\partial \dot{x}_{n}}=\frac{\pi \eta}{T}|n| \dot{x}_{n}, \quad p_{n}^{y} \equiv \frac{\partial L}{\partial \dot{y}_{n}}=\frac{\pi \eta}{T}|n| \dot{y}_{n},
$$

and the corresponding "Hamiltonian" is

$$
\begin{aligned}
H & =\frac{1}{2 \pi} \sum_{n \neq 0,1}\left[\frac{T}{|n| \eta}\left(\left(p_{n}^{x}\right)^{2}+\left(p_{n}^{y}\right)^{2}\right)+\frac{(2 \pi)^{2} R \lambda^{2}}{T}\left(x_{n}^{2}+y_{n}^{2}\right)\right] \\
& =\left(\frac{4 R T \lambda^{2}}{\eta}\right)^{\frac{1}{2}} \sum_{n \neq 0,1} \frac{1}{|n|^{\frac{1}{2}}}\left[\left(a_{n}^{x}\right)^{\dagger} a_{n}^{x}+\left(a_{n}^{y}\right)^{\dagger} a_{n}^{y}+1\right] .
\end{aligned}
$$

Here, the creation and annhilation operators $\left(a_{n}^{x}\right)^{\dagger},\left(a_{n}^{y}\right)^{\dagger}, a_{n}^{x}$ and $a_{n}^{y}$, are defined by

$$
\begin{aligned}
\left(a_{n}^{x}\right)^{\dagger} & =\sqrt{\frac{T}{4 \pi R^{\frac{1}{2}} \lambda \eta^{\frac{1}{2}}|n|^{\frac{1}{2}}}}\left\{p_{n}^{x}+i\left(\frac{4 \pi^{2} R \lambda^{2} \eta|n|}{T}\right)^{\frac{1}{2}} x_{n}\right\}, \\
a_{n}^{x} & =\sqrt{\frac{T}{4 \pi R^{\frac{1}{2}} \lambda \eta^{\frac{1}{2}}|n|^{\frac{1}{2}}}}\left\{p_{n}^{x}-i\left(\frac{4 \pi^{2} R \lambda^{2} \eta|n|}{T}\right)^{\frac{1}{2}} x_{n}\right\}, \\
\left(a_{n}^{y}\right)^{\dagger} & =\sqrt{\frac{T}{4 \pi R^{\frac{1}{2}} \lambda \eta^{\frac{1}{2}}|n|^{\frac{1}{2}}}}\left\{p_{n}^{y}+i\left(\frac{4 \pi^{2} R \lambda^{2} \eta|n|}{T}\right)^{\frac{1}{2}} y_{n}\right\}, \\
a_{n}^{y} & =\sqrt{\frac{T}{4 \pi R^{\frac{1}{2}} \lambda \eta^{\frac{1}{2}}|n|^{\frac{1}{2}}}}\left\{p_{n}^{y}-i\left(\frac{4 \pi^{2} R \lambda^{2} \eta|n|}{T}\right)^{\frac{1}{2}} y_{n}\right\} .
\end{aligned}
$$


We conclude that the zero-point energy can be evaluated by means of the formula

$$
E_{0}=\left(\frac{4 R T \lambda^{2}}{\eta}\right)^{\frac{1}{2}} \sum_{n \neq 0,1} \frac{1}{|n|^{\frac{1}{2}}}=\left(\frac{4 R T \lambda^{2}}{\eta}\right)^{\frac{1}{2}}[2 \zeta(-1 / 2)-1] .
$$

In the second line of Eq. (74) we have used the definition of the zeta function $\zeta(x)$. Note that the apparent divergence in the zero-point energy comes from the infrared sector. In fact, the contribution to the zero-point energy of the mode $n$ decreases, as $|n|$ increases. This corresponds to the fact that the efficiency of the translation or rotation of the micro-organism becomes better for higher modes [2]. And these infinitely decreasing zero-point energies pile up to an infrared divergence but no contribution from an ultraviolet one appears.

The system of micro-organism has a symmetry of the reparametrization of $\sigma$ in Eq.(59) although there is not a reparametrization symmetry of the time $t$. It should be noted that the zeta-function regularization is the regularization which keeps any symmetry by construction since the Hamiltonian itself is used as a regulator in the Hamiltonian formulation. In our case, the regularization corresponds to the replacement

$$
\begin{aligned}
Z(\sigma) & \rightarrow Z^{s}(\sigma) \\
& \equiv \frac{2}{\pi \Gamma\left(s+\frac{1}{2}\right)} \int_{0}^{\infty} d t \int_{-\infty}^{\infty} d u \int_{-\infty}^{\infty} d v t^{2 s} \mathrm{e}^{-v^{2}+2 i u t} \\
\bar{Z}(\sigma) & \rightarrow \mathrm{e}^{2 i u v\left(\frac{4 R T \lambda^{2}}{\eta}\right)^{-\frac{1}{2}} H} Z(\sigma) \mathrm{e}^{-2 i u v\left(\frac{4 R T \lambda^{2}}{\eta}\right)^{-\frac{1}{2}} H} \\
\left(a_{n}^{x, y},\left(a_{n}^{x, y}\right)^{\dagger}\right. & \left.\rightarrow|n|^{-s} a_{n}^{x, y},|n|^{-s}\left(a_{n}^{x, y}\right)^{\dagger}\right)
\end{aligned}
$$

Therefore the terms dropped by the analytic continuation of $s$ do not break any symmetry.

\section{Summary.}

The swimming of micro-organisms in a fluid and the motion of strings (and membranes) in background fields have many points in common. If the surface of a micro-organism is viewed as a string (or membrane), its vibration (the swimming motion) induces a flow of the fluid. The thus induced flow can affect the motion of another micro-organism (another string or membrane), what results in an interaction between the two micro-organisms, or the motion of the original micro-organism itself, originating a self-interaction. Therefore, the mutual and self-interactions between micro-organisms (represented by strings or membranes) are mediated by the velocity field of the fluid.

It is well-known that the interactions between strings or membranes can be mediated by various background fields, such as the graviton, anti-symmetric fields and gauge fields. In these theories, the study of how to estimate the static potential (the Casimir energy) of strings and membranes has been developed extensively, incorporating quantum effects by using, e.g., the zeta function method. Here, our aim has been to apply recently developed techniques in string and membrane theories to the basic problem of micro-organisms' swimming.

In sections 2 and 3 we have carried out the analysis of the static potential for strings and membranes under a constant magnetic field, in the example of the background field $U(1)$, a gauge field, where the existence of the magnetic field changes the strength of the 
Coulomb potential. We have summarized the effect of the anti-symmetric field to the static potential of the string, where by integrating out the anti-symmetric fields one introduces the renormalization effect on the string tension. The case of the coupling with antisymmetric fields has been summarized in Sect. 4.

Using this sound theoretical background, we have studied the swimming motion of ciliates in a two dimensional fluid. The statistical weight of the fluid dynamics is given by $e^{S}$ with its entropy $S$ (in units of the Bolzmann constant $k_{B}=1$ ), so that the "Lagrangean" $L$ of the system should be $-\dot{S}$. It is interesting to notice that the "Lagrangean" $L$ of our incompressible fluid is identical to the action of QED in the Landau gauge, if the velocity field $v_{\mu}(x)$ is recognized as a $U(1)$ gauge field (see Eq. (58)). Introduction of the micro-organism, with its surface parametrized by $X^{\mu}(\xi)$, requires the no-slipping condition: $\dot{X}^{\mu}(\xi)=\left.v^{\mu}(x)\right|_{x=X(\xi)}$. Then, the gauge field $v_{\mu}(x)$ can mediate the Coulomb interaction between the non-local momenta $P_{\mu}(\xi)$, the Fourier transform of $\dot{X}^{\mu}(\xi)$ (see Eqs. (60) and (61)).

This situation of mediating a Coulomb-like interaction between non-local objects can be commonly found in string (and membrane) theories coupled with background fields. Coupling of the gauge fields $A_{\mu}(x)$ induces a Coulomb interaction between the non-local currents $\partial_{i} X^{\mu}(\xi)(i=1$ in Eq. (1) and $i=1,2$ in Eq. (17)), whereas the antisymmetric field $A_{\mu \nu}(x)$ induces the same interaction between the non-local surface elements $\sigma^{\mu \nu}(\xi)=$ $\epsilon^{i j} \partial_{i} X^{\mu}(\xi) \partial_{j} X^{\nu}(\xi)$ (see (37)).

When we prepare two different micro-organisms, strings or membranes, a Coulomb-like interaction between them is generated. This interaction is very important for the description of phenomena involving the collective motion of the micro-organisms, as is the case of the red tide [5]. Specifically, in this paper we have discussed in detail the self-interactions by taking up a single micro-organism, string or membrane, the non-local configuration of which brings about an interesting behavior on the static potential and on the Casimir energy. The zero-point energy of the micro-organism is found to be smaller for the higher vibration modes in the ciliate swimming motion, what corresponds to the fact that the efficiency or power becomes better for the higher modes.

In the calculation of the Casimir energy of the micro-organism we have found infrared divergences rather than ultraviolet ones (see Eq. (74)). The reason for this interchange that occurs between the ultraviolet and the infrared regions may be understood from the fact that in the micro-organisms' swimming, the Coulomb interaction works between the momentum $P^{\mu}(\xi)$ 's and not between the $\dot{X}^{\mu}(\xi)$ 's. The former and the latter are Fourier conjugate with each other, so that the ultraviolet behavior of $P^{\mu}(\xi)$ corresponds to the infrared behavior of $\dot{X}^{\mu}(\xi)$. For such a discussion, the swimming problem of micro-organisms in a two (resp. three) dimensional fluid can be dually related with the string (resp. membrane) theory coupled with the $U(1)$ gauge field by means of the current $\dot{X}^{\mu}(\xi)$.

Acknowledgments. We thank O. Alvarez for discussions on our approach. This work has been supported by DGICYT (Spain), projects PB93-0035 and SAB93-0024, by CIRIT (Generalitat de Catalunya), and by ISF, project RI1000. 


\section{References}

[1] A. Shapere and F. Wilzcek, J. Fluid Mech. 198 (1989) 557.

[2] A. Shapere and F. Wilzcek, J. Fluid Mech. 198 (1989) 587.

[3] M. Kawamura, A. Sugamoto, and S. Nojiri, Mod. Phys. Lett. A9 (1994) 1159; Swimming of Micro-organism and the String- and Membrane-like Algebra, preprint OCHA-PP-43, NDA-FP-15 (1994) (contributed paper to ICHEP 94) hep-th/9406109.

[4] S. Nojiri, M. Kawamura and A. Sugamoto, Phys. Lett. B343 (1995) 181.

[5] S. Nojiri, M. Kawamura, and A. Sugamoto, Collective Motion of Micro-organism from Field Theoretical Viewpoint, preprint NDA-FP-21, OCHA-PP-65 (1995) hepth/9508112

[6] E. Elizalde and S.D. Odintsov, Bosonic string with antisymmetric fields and a non-local Casimir effect, Barcelona preprint, hep-th/9506061 (1995).

[7] V. Nesterenko, Int. J. Mod. Phys. A4 (1989) 2627; E. Ferrer, E.S. Fradkin and V. de la Incera, Phys. Lett. B248 (1990) 281; I.M. Lichtzier S.D. Odintsov and A.A. Bytsenko, Acta Phys. Polon. B22 (1991) 761; J.G. Russo and L. Susskind, Nucl. Phys. B (1995).

[8] O. Alvarez, Phys. Rev. D24 (1981) 440.

[9] M. Lüscher, K. Symanzik and P. Weisz, Nucl. Phys. B173 (1980) 365.

[10] E. Elizalde, S.D. Odintsov, A. Romeo, A.A. Bytsenko and S. Zerbini, Zeta regularization techniques with applications (World Sci., Singapore, 1994).

[11] E. Elizalde, Nuovo Cim. 104B (1989) 685; E. Elizalde, J. Math. Phys. 35 (1994) 3308 and 6000 .

[12] P.S. Howe, R.W. Tucker, J. Phys. A10 (1977) L155; A. Sugamoto, Nucl. Phys. B215 (1983) 381.

[13] S. Chowla and A. Selberg, Proc. Nat. Acad. Sci. US 35 (1949) 317.

[14] E. Elizalde, J. Phys. A27 (1994) 3775.

[15] E. Elizalde, Explicit analytical continuation of the inhomogeneous epstein zeta function, Hiroshima Univ. preprint (1994).

[16] J. Polchinski and Z. Yang, Phys. Rev. D46 (1992) 3667.

[17] G. German and H. Kleinert, Phys. Lett. B225 (1989) 107.

[18] E. Elizalde, S. Leseduarte and S.D. Odintsov, Phys. Rev. D48 (1993) 1757.

[19] K.G. Wilson, Phys. Rev. D10 (1974) 2445. 
[20] G. 't Hooft, Nucl. Phys. B72 (1974) 461.

[21] H. Kleinert, Phys. Lett. B174 (1986) 335; A.M. Polyakov, Nucl. Phys. B268 (1986) 406.

[22] M. Awada and D. Zoller, Phys. Lett. B325 (1994) 115.

[23] S.W. Hawking, Commun. Math Phys. 55 (1977) 133; J.S. Dowker and R. Critchley, Phys. Rev. D13 (1976) 3224; L.S. Brown and G.J. MacLay, Phys. Rev. 184 (1969) 1272; L. Brink and H.B. Nielsen, Phys. Lett. B45 (1973) 332.

[24] J.S. Dowker, Commun. Math. Phys. 162 (1994) 633; J.S. Dowker and J.S. Apps, Manchester preprint MUTP/95/1 (1995). 